\title{
Cooperative diversity using per-user power control in the multiuser MAC channel
}

\author{
Kamel Tourki \\ Laboratoire I3S CNRS UNSA \\ 2000, rte des Lucioles BP 121 \\ 06903 Sophia Antipolis Cedex \\ Email: tourki@i3s.unice.fr
}

\author{
David Gesbert \\ Institut Eurecom \\ 2229, rte des Cretes BP 193 \\ 06904 Sophia Antipolis Cedex \\ Email: david.gesbert@eurecom.fr
}

\author{
Luc Deneire \\ Laboratoire I3S CNRS UNSA \\ 2000, rte des Lucioles BP 121 \\ 06903 Sophia Antipolis Cedex \\ Email: deneire@i3s.unice.fr
}

\begin{abstract}
We consider a multiuser MAC fading channel with two users communicating with a common destination, where each user mutually acts as a relay for the other one as well as transmits his own information. We propose a power control-enhanced cooperative transmission scheme allowing each user to allocate a certain amount of power for his own transmitted data while the rest is devoted to relaying. The underlying protocol is based on a modification of the so-called non-orthogonal amplify and forward (NAF) protocol [1]. We develop capacity expressions for our scheme and derive the rate-optimum power allocation, in closed form. Our results indicate that even in a mutual cooperation setting like ours, on any given realization of the channel, one of the users will always allocate zero power to relaying the data of the other one.
\end{abstract}

\section{INTRODUCTION}

In many wireless applications, wireless users may not be able to support multiple antennas due to size, complexity, power, or other constraints. The wireless medium brings along its unique challenges such as fading and multiuser interference, which can be combatted via the concept of cooperative diversity [2]-[4]. In traditional cooperative diversity setups, a user is unilaterally designated to act as a relay for the benefit of another one, at least for a given period of time. In multiuser networks however, there will be a compromise to strike by all users between transmitting their own information and helping others by relaying their data to the destination. A simplified instance of this scenario is given by a multiple access channel with two or more users trying to reach a common destination (e.g. base station). Since each user wishes to send its own information, it must allocate resource (the total of which is constrained at each user) wisely between its own data transmission and the data it will relay. In this paper we consider resource control in the form of power allocated by a user across its own data and its relay data. The underlying protocol considered here is similar to the one considered by Azarian et al. in [1], which itself evolved from the early work by Laneman, Tse, and Wornell [2]. There, the authors imposed the half-duplex constraint on the cooperating nodes and proposed several cooperative transmission protocols. All the proposed schemes in [2] used a time-division multipleaccess (TDMA) strategy, where the two partners relied on the use of orthogonal signaling to repeat each other's signals. Hence in this work relay and own transmission operations take place in orthogonal resource slots. Recently non-orthogonal signaling strategies have been proposed, e.g. [1], in which a relay transmits delayed information by a user while this user simultaneously transmit fresh data. In this non-orthogonal amplify-forward (NAF) scheme, the diversity-multiplexing trade-off is studied, showing the superiority of the NAF scheme over the orthogonal counterpart. However in [1] and much previous work, the relay network model is unbalanced in the sense that the transmission of own data by the relay is not considered, and the source node is not invited to act as a relay either. In multiuser networks, it is desirable from a global capacity point of view that each user allocates a fraction of its resource toward cooperation.

In this paper we consider a cooperative diversity setup based on a modified NAF protocol, with two users and a common destination (cooperative MAC channel). Each user is allocated a total resource in terms of its average transmit power which it can distribute toward the transmission of its own information and the data it relays for the other user. We consider the problem of maximizing the sum rate for this cooperative MAC channel, as function of the power allocation toward own and relay data, given the knowledge of the channel for both users. We derive the optimum power allocation policy in closed form. In this policy the user with instantaneously better channel conditions (in a sense defined later) is the one for which help is requested. We show that in fact, when the optimum policy is used, one of the users always acts completely selfishingly. Interestingly, this type of selfish behavior by some users in multiuser cooperative MAC was noted by [5], but in a different context with decode-and-forward signaling.

Notations: All boldface letters indicate vectors (lower case) or matrices (upper case). The operator $\operatorname{det}()$ is the determinant of matrix, with ()$^{H}$ denoting its conjugate-transpose and ( )* denoting its conjugate. $\mathbf{E}[$.$] is the expectation operator.$

\section{System MOdEL}

We consider a two user fading Gaussian Multiple Access Channel (MAC), where both the receiver and the transmitters receive noisy versions of the transmitted messages. Each receiver maintains channel state information and employs coherent detection. The channels between users (inter-user channels) and from each user to the destination (uplink channels) are 
TABLE I

POWER ALLOCATION COEFFICIENTS OVER TWO FRAMES FOR 2 USERS TRANSMITTING TO A BASE USING TDMA SCHEME. POWER LEVELS ARE USED TO EITHER SEND OWN OR RELAY DATA. $T_{1}$ (RESP. $T_{2}$ ) IS FIRST (RESP. SECOND) HALF OF THE FRAME.

\begin{tabular}{|c||c|c||c|c|}
\hline & $T_{1}$ & $T_{2}$ & $T_{1}$ & $T_{2}$ \\
\hline user 1 & 1 & $1-\alpha$ & 0 & $\alpha$ \\
user 2 & 0 & $\beta$ & 1 & $1-\beta$ \\
\hline
\end{tabular}

mutually independent. Time is divided in two consecutive frames. Each frame is further divided in two half-frames $T_{1}$ and $T_{2}$. We use a combination of TDMA and non-orthogonal signaling: In the first half of frame 1, user 1 sends its first half packet (containing $\frac{N}{2}$ bits) while user 2 listens. In the second half, user 2 relays the overheard data with power level $\beta$, while user 1 simultaneously sends fresh information (its second half packet) with power level $1-\alpha$. In frame 2 , the roles of user 1 and $2, \alpha, \beta$ are reversed. Thus we maintain a constant average power across the two frames, for each user, regardless of the choice of $\alpha, \beta$.

\section{A. Signal model}

The signal received by the common destination during the first frame (first and second half) is given by,

$$
\left\{\begin{array}{c}
y_{1}(n)=h_{01} x_{1}(n)+z_{0}(n) \\
y_{1}\left(n+\frac{N}{2}\right)=\sqrt{1-\alpha} h_{01} x_{1}\left(n+\frac{N}{2}\right)+\sqrt{\beta} h_{02} A_{1} \times \\
{\left[h_{21} x_{1}(n)+w_{2}(n)\right]+z_{0}\left(n+\frac{N}{2}\right)}
\end{array}\right.
$$

During the second frame, the received signal is:

$$
\left\{\begin{array}{c}
y_{2}(n)=h_{02} x_{2}(n)+z_{0}(n) \\
y_{2}\left(n+\frac{N}{2}\right)=\sqrt{1-\beta} h_{02} x_{2}\left(n+\frac{N}{2}\right)+\sqrt{\alpha} h_{01} A_{2} \times \\
{\left[h_{12} x_{2}(n)+w_{1}(n)\right]+z_{0}\left(n+\frac{N}{2}\right)}
\end{array}\right.
$$

where $n=1, . ., \frac{N}{2}$ and $h_{i j}$ captures the effects of fading between transmitter $j$ and receiver $i$.

Thus, in (1) and (2), $\alpha$ and $\beta$ can be seen as cooperation levels for user 1 and user 2 respectively. $x_{j \in\{1,2\}}(n) \in \mathcal{C}$ is the $n^{t h}$ source symbol, $w_{i \in\{1,2\}}(n)$ and $z_{0}(n)$ are respectively the noise sample (of variance $N_{i \in\{1,2\}}$ ) observed by the transmitter $j \in\{1,2\}$ and the noise sample (of variance $N_{0}$ ) observed by the destination. $h_{21}$ and $h_{12}$ represent the interuser channel gains, and $h_{01}$ and $h_{02}$ denote the user-destination channel gains, which are maintained constant during $T_{1}+T_{2}$. $A_{1} \leq \sqrt{\frac{P_{2}}{\left|h_{21}\right|^{2} P_{1}+N_{2}}}$ and $A_{2} \leq \sqrt{\frac{P_{1}}{\left|h_{12}\right|^{2} P_{2}+N_{1}}}$ are the relay repetition gains, where $P_{j \in\{1,2\}}$ is the sample energy. We remark that (1) and (2) are reduced to equations of an orthogonal direct transmission (non-cooperative protocol) if $\alpha=\beta=0$, and to an amplify-and-forward protocol if $\alpha=\beta=1[2]$.

\section{ANALYSIS OF SUM RATE}

In the proposition below, we develop the expression for the sum rate for the above protocol and power allocation system in a way similar to developments by Laneman et al. and others. a) Proposition 1: For the Gaussian memoryless multipleaccess channel with user cooperation, if the rate pair $(\mathrm{R} 1, \mathrm{R} 2)$ is achievable, then the sum-rate $R 1+R 2 \leq I_{\alpha, \beta}$ where

$$
\begin{aligned}
I_{\alpha, \beta} & \triangleq \log _{2}\left[1+\gamma_{01}+(1-\alpha) \frac{K_{1}}{l_{1}(\beta)}+f\left(\beta \gamma_{02}, \gamma_{21}\right)\right] \\
& +\log _{2}\left[1+\gamma_{02}+(1-\beta) \frac{K_{2}}{l_{2}(\alpha)}+f\left(\alpha \gamma_{01}, \gamma_{12}\right)\right]
\end{aligned}
$$

where

$$
\begin{aligned}
& K_{1}=\left[\gamma_{01}^{2}+\gamma_{01}\right]\left[\gamma_{21}+1\right] \\
& K_{2}=\left[\gamma_{02}^{2}+\gamma_{02}\right]\left[\gamma_{12}+1\right] \\
& l_{1}(\beta)=1+\gamma_{21}+\beta \gamma_{02} \\
& l_{2}(\alpha)=1+\gamma_{12}+\alpha \gamma_{01} \\
& f(x, y)=\frac{x y}{x+y+1}
\end{aligned}
$$

and $\gamma_{i j}$ is defined as $\left|h_{i j}\right|^{2} \frac{P_{j}}{N_{i}}$ where $P_{j}$ is the power of the transmitted signal from user $j, N_{i}$ is the noise power at the receiver $i$ and $i, j \in\{1,2\}$.

Proof: Please refer to the Appendix.

Note that the expression above requires channel information at the receiver but not the transmitter. However the optimization with respect to power control coefficients $\alpha, \beta$ will require full channel knowledge. Extensions of this work to the case of partial knowledge will be reported in a separate contribution. We can consider in the sequel that $P_{1}=P_{2}=P$ and $\gamma_{21}=$ $\gamma_{12}=\gamma$ since the same frequency is used in both directions of inter-user communication.

\section{OPTIMIZATION OF RELAY POWER ALLOCATION}

We now address the problem of optimizing the power allocated by each user toward either transmission of its own data or relay data. The objective function taken here is the multiuser sum rate. We start by characterizing the sum rate in some border points of the power region. The lemma below comes handy in the more general characterization of the optimal power allocation policy.

b) Lemma 1: We characterize the particular expressions of the sum-rate as

$$
\begin{cases}I_{\alpha, 0}>I_{\alpha, 1} & \forall \alpha \\ I_{0, \beta}>I_{1, \beta} & \forall \beta\end{cases}
$$

The proof [6] is omitted here due to the paper size limitation.

\section{A. Power Allocation Algorithm}

The proposition below gives a complete characterization of the optimal power allocation policy for an arbitrary realization of the multiuser channels.

c) Proposition 2: The optimal power allocation which maximizes the sum-rate (3) is given by,

1) $\alpha=\alpha_{*} \neq 0$ and $\beta=0$ if $\left\{\begin{array}{l}\gamma>\gamma_{02}^{2}+\gamma_{02} \\ \gamma_{01}>\frac{\left(1+\gamma_{02}\right)^{2}(1+\gamma)}{\gamma-\left(\gamma_{02}^{2}+\gamma_{02}\right)}-1\end{array}\right.$
2) $\alpha=0$ and $\beta=\beta_{*} \neq 0$ if $\left\{\begin{array}{l}\gamma>\gamma_{01}^{2}+\gamma_{01} \\ \gamma_{02}>\frac{\left(1+\gamma_{01}\right)^{2}(1+\gamma)}{\gamma-\left(\gamma_{01}^{2}+\gamma_{01}\right)}-1\end{array}\right.$

3) $\alpha=0$ and $\beta=0$ if neither condition above is met. 
where optimal values $\alpha_{*}, \beta_{*}$ are detailed in the appendix, and shown below.

Proof: Please refer to the Appendix.

Interpretations: We remark that zero or at most one user out of the two cooperates with the other one. Hence the two users will never both take the role of relay on a given channel realization. In fact the user with "worse" channel conditions always acts selfishly and concentrates all its power for its own data.

\section{B. Power allocation algorithm}

The implementation of the algorithm below requires a centralized power allocation procedure done by e.g. the base. The following intermediate quantities are computed: $A_{1}=$ $K_{1} \gamma_{01}^{2}\left(1+\gamma+\gamma_{02}\right) ; A_{2}=K_{1} \gamma_{01}(1+\gamma)\left(1+\gamma+\gamma_{02}\right)$ $C=K_{1}\left[\gamma \frac{K_{1}}{\gamma_{01}}-\frac{K_{2}}{\gamma_{02}}(1+\gamma)-K_{2}\left(2+\gamma+\gamma_{01}\right)\right]$ $A_{1}^{\prime}=K_{2} \gamma_{02}^{2}\left(1+\gamma+\gamma_{01}\right) ; A_{2}^{\prime}=K_{2} \gamma_{02}(1+\gamma)\left(1+\gamma+\gamma_{01}\right)$ $C^{\prime}=K_{2}\left[\gamma \frac{K_{2}}{\gamma_{02}}-\frac{K_{1}}{\gamma_{01}}(1+\gamma)-K_{1}\left(2+\gamma+\gamma_{02}\right)\right]$

$\operatorname{cond} 1=\frac{K_{2}}{1+\gamma} ; \operatorname{cond} 1=\frac{K_{1}}{1+\gamma} ; \operatorname{cond} 2=\frac{\left(1+\gamma_{02}\right)^{2}(1+\gamma)}{\gamma-\left(\gamma_{02}^{2}+\gamma_{02}\right)}-1$ and condp $2=\frac{\left(1+\gamma_{01}\right)^{2}(1+\gamma)}{\gamma-\left(\gamma_{01}^{2}+\gamma_{01}\right)}-1$.

if $\gamma>$ cond $1 \& \gamma_{01}>$ cond 2 , then

$\alpha_{*}=-\frac{A_{2}}{A_{1}}+\sqrt{\frac{C}{A_{1}}+\left(\frac{A_{2}}{A_{1}}\right)^{2}}$

Decision : user 1 cooperates with a level given by $\alpha_{*} \%$ and resulting in a sum-rate of $I_{\alpha_{*}, 0}$.

else if $\gamma>$ condp $1 \& \gamma_{02}>$ condp2, then

$$
\beta_{*}=-\frac{A_{2}^{\prime}}{A_{1}^{\prime}}+\sqrt{\frac{C^{\prime}}{A_{1}^{\prime}}+\left(\frac{A_{2}^{\prime}}{A_{1}^{\prime}}\right)^{2}}
$$

Decision : user 2 cooperates with a level given by $\beta_{*} \%$ and resulting in a sum-rate of $I_{0, \beta_{*}}$.

else

Decision : No cooperation, $\left[\alpha_{*}, \beta_{*}\right]=[0,0]$, sum-rate $=I_{0,0}$. end if; end if;

\section{Simulation RESUlts}

\section{A. Network Geometry}

We anticipate that cooperation will perform differently as function of the positions of the users wrt destination. Hence we study two different network geometries, denoted by symmetric and asymmetric, see Fig. 1. In the asymmetric case, we model the path-loss, i.e. the mean channel powers $\sigma_{i j}^{2}$, as a function of the relative relay position $r$ without loss of generality by

$$
\sigma_{01}^{2}=1, \sigma_{12}^{2}=d^{-\nu}, \sigma_{02}^{2}=(1-d)^{-\nu}
$$

where $\nu$ is the path loss exponent and $0<d=d_{12}<1$. The distances are normalized by the distance $d_{01}$. In these coordinates, the user 1 can be located at $(0,0)$, and the destination can be located at $(1,0)$, without loss of generality. User 2 is located at $(\mathrm{d}, 0)$ [2]. In the symmetric case, all channels are drawn with same unit-variance.

\section{B. Simulation Results}

We report results for $\nu=4$ and we model all channels as Rayleigh block flat fading with additive white Gaussian noise. Figs. 2-4 show simulated outage capacity behavior for

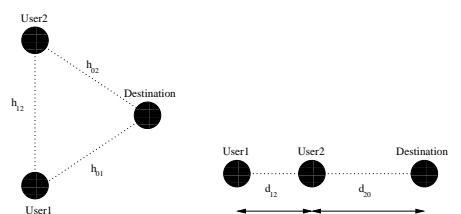

Fig. 1. Symmetric (left) and Asymmetric (right) networks.

the cooperation with optimal power allocation, compared with no cooperation. We look at both single user rate and sum rate performance at 0.1 probability, SNR $=10 \mathrm{~dB}$. In the symmetric case, Fig. 2 shows a marginal improvement in sum rate due to cooperation, due to the fact one user gains from cooperation and the other one suffers by roughly the same amount of data rate. Also the average channel situation with equal instantaneous gains will result in selfish behavior by both users as predicted by our theory (proposition 2).

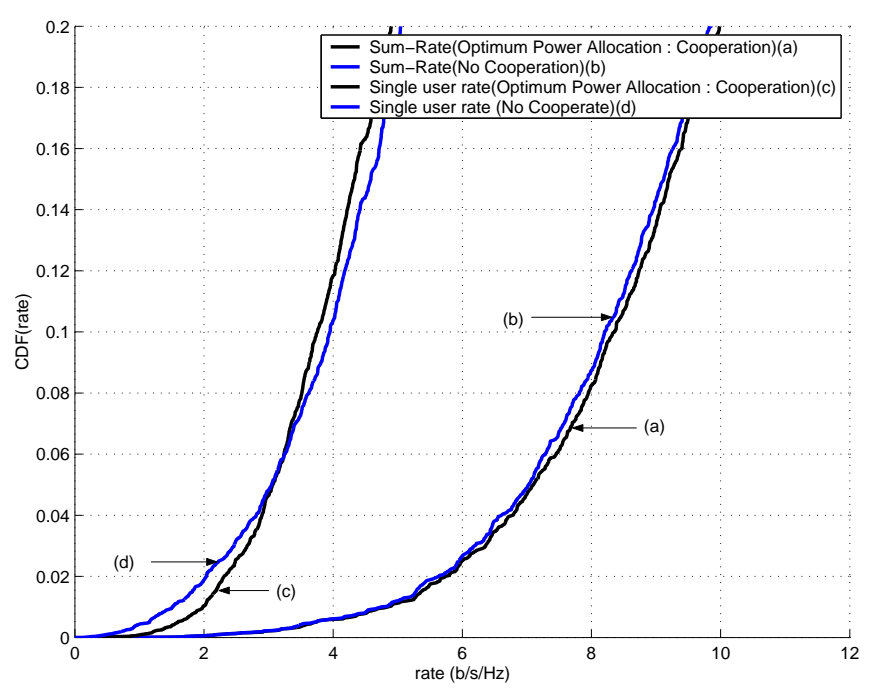

Fig. 2. Simulated outage behavior for symmetric network $\sigma^{2}=1$.

Figs. 3-5 show the simulation results for an asymmetric network when user 2 is located at $(0.1,0),(0.5,0)$ and $(0.9,0)$ respectively. The gains due to optimized cooperation are significant for the user furthers away from the base. However this gain also translates into a sum-rate (system) gain. When $d=0.1$, the sum-rate benefits from cooperation by 0.33 $\mathrm{bit} / \mathrm{s} / \mathrm{Hz}$ and the user 1 benefits by up to $1 \mathrm{bit} / \mathrm{s} / \mathrm{Hz}$. But user 2 which is closer to the destination than user 1 , needs less cooperation.

When user 2 is halfway between user 1 and destination, the sum-rate is ameliorated to $0.75 \mathrm{bit} / \mathrm{s} / \mathrm{Hz}$ due to cooperation, and the user's 1 gain is up to $2 \mathrm{bit} / \mathrm{s} / \mathrm{Hz}$.

But when user 2 is close to the destination, the distance between the users becomes larger, and for an outage probability of $10 \%$, user 1 can not do more than $4 \mathrm{bit} / \mathrm{s} / \mathrm{Hz}$.

\section{CONCLUSION}

We have addressed the problem of optimal power allocation for a fading cooperative MAC, where the users are allowed 


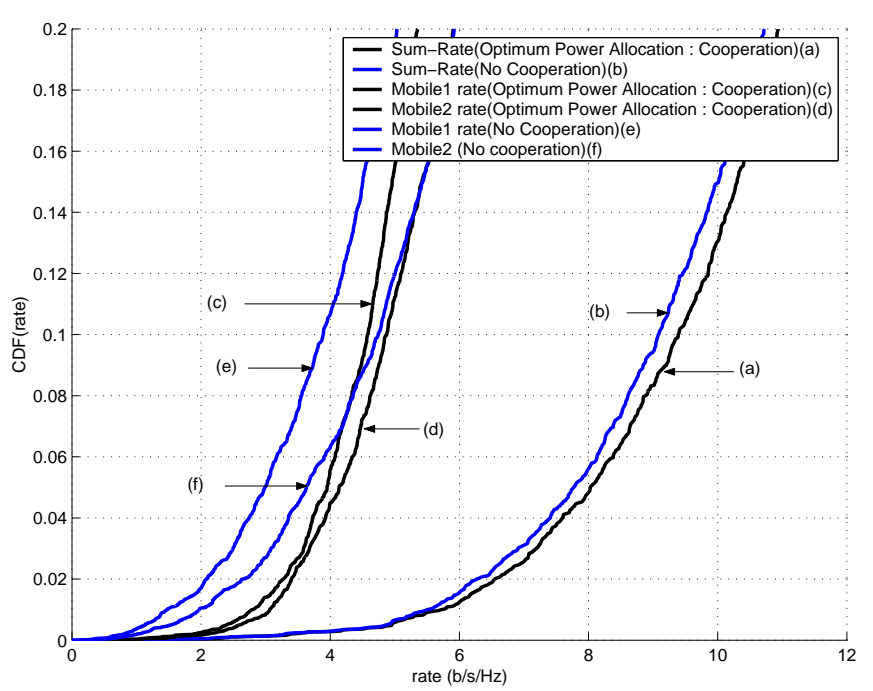

Fig. 3. Simulated outage behavior for Asymmetric network with user 2 located at $(0.1,0)$, i.e, close to the user 1 .

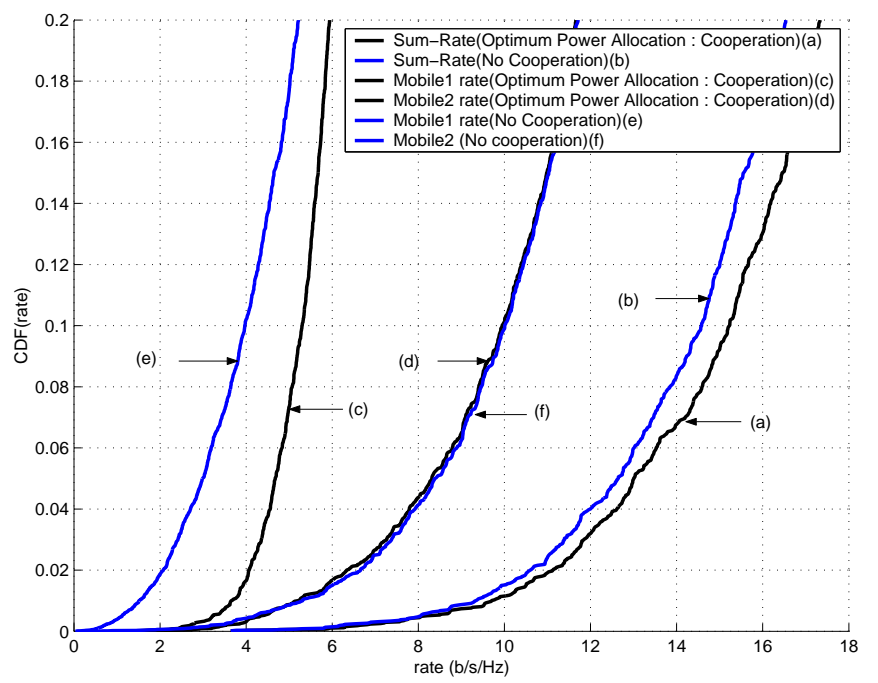

Fig. 4. Simulated outage behavior for Asymmetric network with user 2 located at $(0.5,0)$, i.e, halfway between user 1 and destination.

to adjust the amount of resource spent on own and relay transmission as function of the channel realization. We have characterized the cooperation power levels that maximize the sum-rate. We showed that at most either user 1 cooperates or user 2, but not both, indicating that a selfish behavior for at least one user is optimal from a system perspective.

\section{APPENDIX}

\section{A. Proof of Proposition 1}

For simplicity, and without loss of generality, we formulate (1) as

$$
\underbrace{\left[\begin{array}{c}
y_{1}(n) \\
y_{1}\left(n+\frac{N}{2}\right)
\end{array}\right]}_{\mathbf{y}_{1}}=\underbrace{\left[\begin{array}{cc}
h_{01} & 0 \\
\sqrt{\beta} A_{1} h_{02} h_{21} & \sqrt{1-\alpha} h_{01}
\end{array}\right]}_{\mathbf{M}_{1}} \underbrace{\left[\begin{array}{c}
x_{1}(n) \\
x_{1}\left(n+\frac{N}{2}\right)
\end{array}\right]}_{\mathbf{x}_{1}}
$$

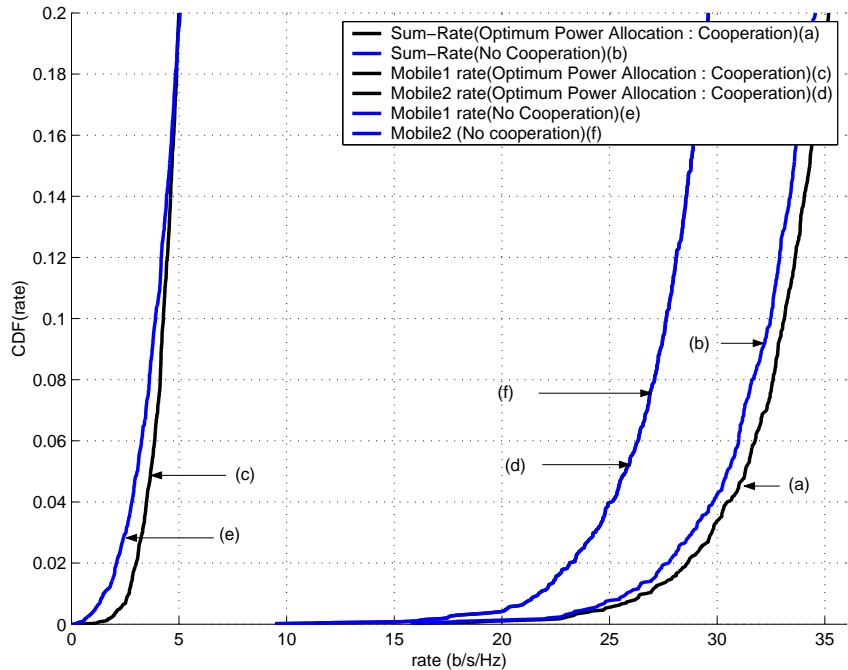

Fig. 5. Simulated outage behavior for Asymmetric network with user 2 located at $(0.9,0)$, i.e, close to the destination.

$$
+\underbrace{\left[\begin{array}{ccc}
0 & 1 & 0 \\
\sqrt{\beta} A_{1} h_{02} & 0 & 1
\end{array}\right]}_{\mathbf{B}_{1}} \underbrace{\left[\begin{array}{c}
w_{2}(n) \\
z_{0}(n) \\
z_{0}\left(n+\frac{N}{2}\right)
\end{array}\right]}_{\mathbf{z}_{1}}
$$

Therefore we compute the maximum average mutual information during $T_{1}+T_{2}$.

$$
\begin{aligned}
I\left(\mathbf{x}_{1}, \widetilde{\mathbf{y}}_{1}\right) & =I\left(\mathbf{x}_{1} ; \mathbf{M}_{1}\right)+I\left(\mathbf{x}_{1} ; \mathbf{y}_{1} / \mathbf{M}_{1}\right) \\
& =I\left(\mathbf{x}_{1} ; \mathbf{y}_{1} / \mathbf{M}_{1}\right) \\
& \leq \log _{2} \operatorname{det}\left(\mathbf{I}_{2}+\mathbf{M}_{1} \mathbf{\Lambda}_{\mathbf{x}_{1}} \mathbf{M}_{1}^{H} \boldsymbol{\Sigma}_{\mathbf{n}_{1}}^{-1}\right)
\end{aligned}
$$

where $\mathbf{n}_{1}=\mathbf{B}_{1} \mathbf{z}_{1}$ and $\boldsymbol{\Lambda}_{\mathbf{x}_{1}}=\mathbf{E}\left(\mathbf{x}_{1} \mathbf{x}_{1}^{H}\right)=P_{1} \mathbf{I}_{2}$.

Therefore, $\boldsymbol{\Sigma}_{\mathbf{n}_{1}}=\mathbf{B}_{1} \mathbf{E}\left(\mathbf{z}_{1} \mathbf{z}_{1}^{H}\right) \mathbf{B}_{1}^{H}$ and equal to

$$
\begin{gathered}
\boldsymbol{\Sigma}_{\mathbf{n}_{1}}=\left[\begin{array}{cc}
N_{0} & 0 \\
0 & N_{0}+\beta\left(A_{1}\right)^{2}\left|h_{02}\right|^{2} N_{2}
\end{array}\right] \\
\mathbf{M}_{1} \mathbf{M}_{1}^{H}=\left[\begin{array}{cc}
A & B \\
C & D
\end{array}\right]
\end{gathered}
$$

where

$$
\begin{aligned}
& A=\left|h_{01}\right|^{2} \\
& B=\sqrt{\beta} A_{1} h_{01}\left(h_{02}\right)^{*}\left(h_{21}\right)^{*} \\
& C=\sqrt{\beta} A_{1}\left(h_{01}\right)^{*} h_{02} h_{21} \\
& D=(1-\alpha)\left|h_{01}\right|^{2}+\beta\left(A_{1}\right)^{2}\left|h_{02} h_{21}\right|^{2}
\end{aligned}
$$

and after substitutions and algebraic manipulations, we obtain

$$
\begin{aligned}
& \log _{2} \operatorname{det}\left(\mathbf{I}_{2}+P_{1} \mathbf{M}_{1} \mathbf{M}_{1}^{H} \boldsymbol{\Sigma}_{\mathbf{n}_{1}}^{-1}\right)=\log _{2}\left[1+\gamma_{01}\right. \\
& \left.+(1-\alpha) \frac{K_{1}}{l_{1}(\beta)}+f\left(\beta \gamma_{02}, \gamma_{21}\right)\right]
\end{aligned}
$$

so, (3) is straightforward.

\section{B. Proof of Proposition 2}

In order to seek $\left(\alpha_{*}, \beta_{*}\right)$ for which $I_{\alpha, \beta}$ is maximized,

$$
\left(\alpha_{*}, \beta_{*}\right)=\arg \max _{\alpha, \beta \in[0,1]} I_{\alpha, \beta}
$$


we must solve this system of equations :

$$
\left\{\begin{array}{l}
\frac{\partial I_{\alpha, \beta}}{\partial \partial_{\alpha, \beta}}=0 \\
\frac{\partial \beta}{\partial \beta}=0
\end{array}\right.
$$

The partial derivatives of $I_{\alpha, \beta}, \frac{\partial I_{\alpha, \beta}}{\partial \alpha}$ and $\frac{\partial I_{\alpha, \beta}}{\partial \beta}$ respectively to $\alpha$ and $\beta$ give

$$
\begin{aligned}
\frac{\partial I_{\alpha, \beta}}{\partial \alpha}= & \frac{1}{\ln (2)}\left[\frac{\frac{-K_{1}}{l_{1}(\beta)}}{1+\gamma_{01}+(1-\alpha) \frac{K_{1}}{l_{1}(\beta)}+f\left(\beta \gamma_{02}, \gamma\right)}\right. \\
& \left.+\frac{(1-\beta) K_{2} \frac{\frac{\partial l_{2}(\alpha)}{\partial \alpha}}{\left[l_{2}(\alpha)\right]^{2}}+\frac{\gamma \gamma_{01}(1+\gamma)}{\left[l_{2}(\alpha)\right]^{2}}}{1+\gamma_{02}+(1-\beta) \frac{K_{2}}{l_{2}(\alpha)}+f\left(\alpha \gamma_{01}, \gamma\right)}\right]
\end{aligned}
$$

and

$$
\begin{aligned}
\frac{\partial I_{\alpha, \beta}}{\partial \beta}= & \frac{1}{\ln (2)}\left[\frac{\frac{-K_{2}}{l_{2}(\alpha)}}{1+\gamma_{02}+(1-\beta) \frac{K_{2}}{l_{2}(\alpha)}+f\left(\alpha \gamma_{01}, \gamma\right)}\right. \\
& \left.+\frac{(1-\alpha) K_{1} \frac{\frac{-\partial l_{1}(\beta)}{\partial \beta}}{\left[l_{1}(\beta)\right]^{2}}+\frac{\gamma \gamma_{02}(1+\gamma)}{\left[l_{1}(\beta)\right]^{2}}}{1+\gamma_{01}+(1-\alpha) \frac{K_{1}}{l_{1}(\beta)}+f\left(\beta \gamma_{02}, \gamma\right)}\right]
\end{aligned}
$$

after some simplifications, $\alpha_{*}$ and $\beta_{*}$ are determined as solutions of

$$
\left\{\begin{array}{l}
A_{1} \alpha^{2}+2 \alpha A_{2}-C-B_{2} \beta-B_{1} \beta^{2}=0 \\
A_{1}^{\prime} \beta^{2}+2 \beta A_{2}^{\prime}-C^{\prime}-B_{2}^{\prime} \alpha-B_{1}^{\prime} \alpha^{2}=0
\end{array}\right.
$$

where

$$
\begin{aligned}
A_{1}= & K_{1} \gamma_{01}^{2}\left(1+\gamma+\gamma_{02}\right) \\
A_{2}= & K_{1} \gamma_{01}(1+\gamma)\left(1+\gamma+\gamma_{02}\right) \\
B_{1}= & K_{2} \gamma_{01} \gamma_{02}\left(1+\gamma+\gamma_{01}\right) \\
B_{2}= & K_{1} K_{2}\left(2+\gamma+\gamma_{01}\right)+\gamma_{01} \gamma_{02}\left(1+\gamma+\gamma_{01}\right) \times \\
& \quad\left(\gamma(1+\gamma)-K_{2}\right) \\
C= & K_{1}\left[\gamma \frac{K_{1}}{\gamma_{01}}-\frac{K_{2}}{\gamma_{02}}(1+\gamma)-K_{2}\left(2+\gamma+\gamma_{01}\right)\right] \\
A_{1}^{\prime}= & K_{2} \gamma_{02}^{2}\left(1+\gamma+\gamma_{01}\right) \\
A_{2}^{\prime}= & K_{2} \gamma_{02}(1+\gamma)\left(1+\gamma+\gamma_{01}\right) \\
B_{1}^{\prime}= & K_{1} \gamma_{01} \gamma_{02}\left(1+\gamma+\gamma_{02}\right) \\
B_{2}^{\prime}= & K_{1} K_{2}\left(2+\gamma+\gamma_{02}\right)+\gamma_{01} \gamma_{02}\left(1+\gamma+\gamma_{02}\right) \times \\
& \left(\gamma(1+\gamma)-K_{1}\right) \\
C^{\prime}= & K_{2}\left[\gamma \frac{K_{2}}{\gamma_{02}}-\frac{K_{1}}{\gamma_{01}}(1+\gamma)-K_{1}\left(2+\gamma+\gamma_{02}\right)\right]
\end{aligned}
$$

therefore, the system (14) becomes

$$
\left\{\begin{array}{l}
\frac{\widetilde{\alpha}^{2}}{B_{1}}-\frac{\widetilde{\beta}^{2}}{A_{1}}=\kappa_{1} \\
\frac{\widetilde{\beta}^{2}}{B_{1}^{\prime}}-\frac{\widetilde{\alpha}^{2}}{A_{1}^{\prime}}=\kappa_{2}
\end{array}\right.
$$

where

$$
\begin{gathered}
\left\{\begin{array}{l}
\widetilde{\alpha}=\alpha+\frac{A_{2}}{A_{1}} \\
\tilde{\beta}=\beta+\frac{B_{2}}{2 B_{1}}
\end{array}\right. \\
\left\{\begin{array}{l}
\kappa_{1}=\frac{C}{A_{1} B_{1}}+\frac{1}{B_{1}}\left(\frac{A_{2}}{A_{1}}\right)^{2}-\frac{1}{A_{1}}\left(\frac{B_{2}}{2 B_{1}}\right)^{2} \\
\kappa_{2}=\frac{C^{\prime}}{A_{1}^{\prime} B_{1}^{\prime}}+\frac{1}{B_{1}^{\prime}}\left(\frac{A_{2}^{\prime}}{A_{1}^{\prime}}\right)^{2}-\frac{1}{A_{1}^{\prime}}\left(\frac{B_{2}^{\prime}}{2 B_{1}^{\prime}}\right)^{2}
\end{array}\right.
\end{gathered}
$$

In (19), we have two equations of hyperboles. When we replace $\widetilde{\alpha}$ in the second equation by its expression derived from the first one in order to solve this system we obtain

$$
\widetilde{\beta}^{2}\left(\frac{1}{B_{1}^{\prime}}-\frac{A_{1}}{A_{1}^{\prime} B_{1}}\right)=\underbrace{\kappa_{2}+\frac{B_{1}}{A_{1}^{\prime}} \kappa_{1}}_{\neq 0}
$$

and because we have

$$
\frac{B_{1}}{A_{1}}=\frac{A_{1}^{\prime}}{B_{1}^{\prime}}
$$

it is straightforward that there are no solutions, graphically traduced by the no intersection between these hyperboles where eq. (23) shows the equality of the slopes of the asymptotes, unless on the plans $\mathcal{P}_{\alpha, 0}=\{\beta=0, \forall \alpha\}, \mathcal{P}_{\alpha, 1}=\{\beta=1, \forall \alpha\}$, $\mathcal{P}_{0, \beta}=\{\alpha=0, \forall \beta\}$ and $\mathcal{P}_{1, \beta}=\{\alpha=1, \forall \beta\}$.

Using proposition 2 , we are interested only by $I_{\alpha, 0}$ and $I_{0, \beta}$. Therefore at most one user cooperate, so

$$
\left\{\begin{array}{l}
\alpha_{*}=\arg \max _{\alpha \in[0,1]} I_{\alpha, 0} \\
\beta_{*}=\arg \max _{\beta \in[0,1]} I_{0, \beta}
\end{array}\right.
$$

The derivatives of $I_{\alpha, 0}$ and $I_{0, \beta}, \frac{d I_{\alpha, 0}}{d \alpha}$ and $\frac{d I_{0, \beta}}{d \beta}$ give

$$
\left\{\begin{array}{l}
\widetilde{\alpha}^{2}=\frac{C}{A_{1}}+\left(\frac{A_{2}}{A_{1}}\right)^{2} \\
\widetilde{\beta}^{2}=\frac{C^{\prime}}{A_{1}^{\prime}}+\left(\frac{A_{2}^{\prime}}{A_{1}^{\prime}}\right)^{2}
\end{array}\right.
$$

Therefore, $\alpha_{*}$ exists when

$$
\left\{\begin{array}{l}
\frac{C}{A_{1}}+\left(\frac{A_{2}}{A_{1}}\right)^{2}>0 \\
\left.\left.-\left(\frac{A_{2}}{A_{1}}\right)+\sqrt{\frac{C}{A_{1}}+\left(\frac{A_{2}}{A_{1}}\right)^{2}} \in\right] 0,1\right]
\end{array}\right.
$$

and it leads to

$$
\left\{\begin{array}{l}
\gamma>\gamma_{01}^{2}+\gamma_{01} \\
\gamma_{02}>\frac{\left(1+\gamma_{01}\right)^{2}(1+\gamma)}{\gamma-\left(\gamma_{01}^{2}+\gamma_{01}\right)}-1
\end{array}\right.
$$

and the same method is applied to $\beta_{*}$.

\section{REFERENCES}

[1] K. Azarian, H. E. Gamal, and P. Schniter, "On the achievable diversitymultiplexing tradeoff in half-duplex cooperative channels," IEEE Transactions on Informations Theory, vol. 51, no. 12, pp. 4152-4172, December 2005.

[2] J. N. Laneman, D. N. Tse, and G. W. Wornell, "Cooperative diversity in wireless networks: Efficient protocols and outage behavior," IEEE Trans. Inform. Theory, vol. 50, no. 12, pp. 3062-3080, Dec. 2004.

[3] A. Sendonaris, E. Erkip, and B. Aazhang, "User cooperation diversity, parts i,ii," IEEE Trans. Comm., vol. 51, no. 11, pp. 1927-1948, Nov. 2003.

[4] T. M. Cover and C. S. K. Leung, "An achievable rate region for the multiple-access channel with feedback," IEEE Transactions on Informations Theory, vol. IT-27, no. 3, pp. 292-298, May 1981.

[5] O. Kaya and S. Ulukus, "'Power Control for Fading Multiple Access Channels with User Cooperation"," in Proc. IEEE International Conference on Wireless Networks, Communications, and Mobile Computing, Maui, Hawaii, USA, June 2005.

[6] K. Tourki, D. Gesbert, and L. Deneire, "Cooperative diversity using peruser power control in the multiuser mac channel," Journal version in preparation, 2007. 